\title{
Clinical disease and stage of lactation influence shedding of Mycobacterium avium subspecies paratuberculosis into milk and colostrum of naturally infected dairy cows
}

\author{
J. R. Stabel, ${ }^{*} \dagger^{1,2}$ L. Bradner, $\ddagger^{1}$ S. Robbe-Austerman,§ and D. C. Beitz ${ }^{*} \ddagger$ \\ *Department of Animal Science, lowa State University, Ames 50010 \\ tUSDA Agricultural Research Service (ARS), National Animal Disease Center, Ames, IA 50010 \\ ¥Roy J. Carver Department of Biochemistry, Biophysics, and Molecular Biology, lowa State University, Ames 50010 \\ §USDA Animal and Plant Health Inspection Service (APHIS), Veterinary Services, Ames, IA 50010
}

\begin{abstract}
Mycobacterium avium ssp. paratuberculosis (MAP) is the causative agent of Johne's disease (JD). One mode of transmission of MAP is through ingestion of contaminated milk and colostrum by susceptible calves. The objective of this study was to determine if the amount of MAP shed into the milk and colostrum of infected cows was affected by severity of infection as well as the number of days in milk (DIM). Milk was collected over the 305-d lactation period from naturally infected cows in the asymptomatic subclinical $(\mathrm{n}=39)$ and symptomatic clinical $(\mathrm{n}=29)$ stages of disease, as well as 8 noninfected control cows. All milk samples were assayed for MAP by culture on Herrold's egg yolk medium and either BACTEC 12B (Becton Dickinson, Franklin Lakes, NJ) or para-JEM (Thermo Fisher Scientific, Trek Diagnostic Systems Inc., Cleveland, $\mathrm{OH}$ ) liquid medium, and by direct PCR for the IS900 target gene. Mycobacterium avium ssp. paratuberculosis was detected in $3.8,4.1$, and $12.6 \%$ of milk samples collected from cows with subclinical JD after culture in Herrold's egg yolk medium, liquid medium, and direct PCR, respectively. The frequency of MAP positivity increased to $12.9,18.4$, and $49.2 \%$ of milk samples collected from cows with clinical JD by these same methods, respectively. None of the milk samples collected from control cows was positive for MAP by any detection method. Viable MAP was primarily isolated from milk and colostrum of subclinically and clinically infected cows collected in early lactation (DIM 0-60), with negligible positive samples observed in mid (DIM 60-240) and late (DIM 240-305) lactation. This study demonstrates that shedding of MAP into milk is affected by infection status of the cow as well as stage of
\end{abstract}

Received April 3, 2014.

Accepted June 16, 2014.

${ }^{1}$ The first 2 authors contributed equally to this paper.

${ }^{2}$ Corresponding author: judy.stabel@ars.usda.gov lactation, providing useful information to producers to help break the cycle of infection within a herd.

Key words: Mycobacterium avium ssp. paratuberculosis, milk, lactation, shedding

\section{INTRODUCTION}

Johne's disease (JD), caused by Mycobacterium avium ssp. paratuberculosis (MAP), results in significant annual economic loss to the dairy industry, primarily due to reduced milk production and premature culling (Ott et al., 1999). Though primarily transmitted through ingestion of contaminated feces, MAP also can be transmitted through milk and colostrum, as well as in utero (Chiodini et al., 1984). As JD progresses, MAP can disseminate throughout the body and has been isolated from milk, supramammary lymph nodes, and lymph fluid from the udder (Sweeney et al., 1992; Chiodini, 1996; Khol et al., 2012).

Milk and colostrum containing MAP can be consumed by neonates and young stock that are most susceptible to MAP infection (Windsor and Whittington, 2010). Calves that are fed maternal colostrum are more likely to become infected with MAP than those that are fed colostrum replacer or pasteurized colostrum, indicating that raw colostrum and milk may be the initial point of exposure for neonates (Stabel, 2008; Pithua et al., 2009). Furthermore, if producers feed pooled milk or colostrum, they are increasing the likelihood of transmitting MAP to an even larger number of calves. Unfortunately, milk and colostrum replacers are an expensive alternative to feeding pooled milk and colostrum.

Little is known about the factors that influence the amount of MAP shed into milk. The excretion of MAP may be influenced by the stage of infection, with the highest shedding occurring in the clinical stages of JD and very little or even undetectable amounts of MAP shed in early stages (Sweeney et al., 1992). Days in milk also may be correlated with the amount of MAP 
shed into milk and colostrum, because the volume and composition varies greatly throughout the 305-d lactation. Effects of milk volume and composition on the diagnosis of MAP infection using the milk ELISA have been observed (Nielsen and Toft, 2012). A commercial ELISA was used to measure MAP antibodies in over 1 million milk samples collected from cows enrolled in the Danish paratuberculosis control program over a 31-mo period. Results demonstrated that DIM was a significant factor in the identification of positive cows and that early stage lactation samples at 1 to 2 DIM are more likely to test positive, suggesting that dilution factors may be responsible for a loss in sensitivity of detection (Nielsen and Toft, 2012; Zervens et al., 2013). Further testing demonstrated that colostral immunoglobulins were not found to contribute to falsepositive reactions in the ELISA, and measurement of colostral antibody improved the odds of an animal testing positive for MAP infection by 130-fold compared with samples taken at 4 DIM (Zervens et al., 2013). Although the measurement of milk antibodies is quite different than quantifying the shedding of MAP into the milk, these results suggest that DIM may affect the sensitivity of detection. The objectives of this study were to determine if a correlation exists between stage in JD, DIM, and the amount of MAP shed into milk and colostrum of naturally infected dairy cows.

\section{MATERIALS AND METHODS}

\section{Milk Collection}

Milk was collected for 91 complete 305-d lactation cycles over a period of 12 yr from 76 Holstein dairy cows well characterized for JD. Lactation cycles were stratified across infection status of cows as follows: asymptomatic cows with subclinical disease $(\mathrm{n}=39)$ and symptomatic cows with clinical signs of disease ( $\mathrm{n}$ $=29)$, and noninfected control cows $(\mathrm{n}=8)$. Cows were stratified into infection groups by monitoring fecal shedding of MAP by culture and PCR as previously described (Khalifeh et al., 2009). By definition, clinical cows were shedding more than $100 \mathrm{cfu} / \mathrm{g}$ of feces and presented with weight loss and intermittent diarrhea. Subclinically infected cows were shedding less than 10 $\mathrm{cfu} / \mathrm{g}$ of feces and were asymptomatic. The noninfected control cows were characterized by repeated negative fecal culture performed semi-annually over a 3 - to 5-yr period. In addition, these animals were negative for production of antibodies specific for MAP and IFN- $\gamma$ performed during that period.

Due to the long-term collection period, we were able to obtain samples from 2 lactation periods from 13 of the 76 cows and 3 lactation periods from 1 of the 76 cows. Milk was collected into sterile containers after thorough cleaning of the teats on d 1, 3, 7, 14, 21, 28, 90, 180, and 305 after calving. Milk was immediately frozen and stored at $-80^{\circ} \mathrm{C}$ until processing. Animals used in this study were housed at the National Animal Disease Center (NADC, Ames, IA). All procedures performed on the dairy cows were approved by the Institutional Animal Care and Use Committee (NADC).

\section{Positive and Negative Control Milk Preparation}

Milk was obtained from a noninfected healthy cow at the NADC. Milk from the cow was hand stripped into sterile collection containers after thorough disinfection of the udder and teats with iodine teat dip and $70 \%$ ethanol. Large volumes of milk were collected at one time and aliquots were dispensed into tubes and stored at $-80^{\circ} \mathrm{C}$ until use. This milk was used for the negative control and also artificially spiked to a final concentration of $10^{6} \mathrm{cfu} / \mathrm{mL}$ with MAP strain 167 (obtained from a field isolate at the NADC) for the positive control.

\section{Decontamination of Milk with $\mathrm{N}$-Acetyl-L-Cysteine-NaOH}

Each sample of milk was thawed overnight at $4^{\circ} \mathrm{C}$. The samples were lightly shaken to ensure a homogeneous sample. A $20-\mathrm{mL}$ aliquot of the milk was transferred to a sterile container centrifuged at $5,600 \times g$ for $30 \mathrm{~min}$ at $4^{\circ} \mathrm{C}$. The whey layer was discarded, and the cream and pellet layers were retained. The cream and pellet layers were resuspended in PBS (Sigma-Aldrich, St. Louis, MO) to bring the final volume to $5 \mathrm{~mL}$. The samples were decontaminated with $5 \mathrm{~mL}$ of a solution containing sterile $1.45 \%$ sodium citrate, $3.0 \%$ sodium hydroxide, and $0.5 \% \mathrm{~N}$-acetyl-L-cysteine (NALC-3.0\% $\mathrm{NaOH}$; Sigma-Aldrich) as previously described (Bradner et al., 2013). The NALC component of the solution was added immediately before use. The samples were inverted to mix, and incubated for $15 \mathrm{~min}$ at $22^{\circ} \mathrm{C}$. Immediately after incubation, $15 \mathrm{~mL}$ of PBS was added to dilute the $\mathrm{NaOH}$. Samples were centrifuged at 5,600 $\times g$ for $30 \mathrm{~min}$ at $4^{\circ} \mathrm{C}$, the aqueous layer was discarded, and the cream and pellet were resuspended in $1 \mathrm{~mL}$ of PBS for inoculation of the media.

\section{Media for Recovery of MAP}

Initially, BACTEC 12B liquid medium [Becton Dickinson (BD), Franklin Lakes, NJ] and Herrold's egg yolk (HEY) agar medium (BD), were used to culture MAP from milk samples. However, the BACTEC 12B medium was discontinued by the company midway through the experiment and para-JEM medium (Thermo Fisher 
Scientific, Trek Diagnostic Systems Inc., Cleveland, $\mathrm{OH}$ ) was used as an alternative liquid medium for 174 of the 518 samples processed for culture. Briefly, the BACTEC 12B medium was supplemented with 0.5 $\mathrm{mL}$ of unmodified raw egg yolk (in house), $0.2 \mathrm{~mL}$ of PANTA Plus (BD), $0.1 \mathrm{~mL}$ of $50 \mu \mathrm{g} / \mathrm{mL}$ mycobactin J, and $1.2 \mathrm{~mL}$ of sterile deionized water. Each bottle was inoculated with $200 \mu \mathrm{L}$ of decontaminated milk and placed in a $37^{\circ} \mathrm{C}$ incubator. The growth index (GI) of the microorganisms in the bottles was measured by the BACTEC 460 culture system (BD) twice per week for the first $2 \mathrm{wk}$ and then once per week for the following $10 \mathrm{wk}$. The bottles were considered positive when the GI reached 300. Then, they were removed from incubation for MAP confirmation. At the end of the 12-wk incubation period, all bottles were subject to MAP confirmation procedures.

The para-JEM medium was supplemented according to the manufacturer's instructions. Each bottle was inoculated with $500 \mu \mathrm{L}$ of decontaminated milk sample and placed into the ESP Culture System II incubator (Trek Diagnostic Systems Inc.) for up to $84 \mathrm{~d}$ according to the manufacturer's instructions. When the bottles signaled positive and indicated a positive growth curve, the bottles were removed for MAP growth confirmation. At the end of the incubation period, all bottles were subject to MAP growth confirmation procedures noted below. Before the medium was removed from the bottles for growth confirmation, the bottles were shaken on a VXR IKA Vibrax shaker (IKA Works, Inc., Wilmington, NC) for 2 min to dislodge the MAP from the sponges in the bottles. The number of colonyforming units of MAP per milliliter of milk was extrapolated from a standard curve generated by negative milk experimentally inoculated with serial dilutions of MAP strain 167. Triplicate samples of $18 \mathrm{~mL}$ of milk and $2 \mathrm{~mL}$ of the respective dilution of bacterial suspension were prepared to yield final MAP concentrations of $10^{2}, 10^{4}, 10^{6}$, and $10^{8} \mathrm{cfu} / \mathrm{mL}$ of milk (Bradner et al., 2013). Each dilution was subjected to the basic milk processing protocol before culture and days to positive were plotted against MAP concentration to generate a standard curve for each liquid medium.

Mycobacterium avium ssp. paratuberculosis recovery from milk also was evaluated on HEY medium (BD) and compared with BACTEC 12B and para-JEM liquid media. Each slant of HEY medium containing mycobactin $\mathrm{J}$, amphotericin $\mathrm{B}$, nalidixic acid, and vancomycin was inoculated with $100 \mu \mathrm{L}$ of decontaminated milk sample in replicates of 3 agar slants per sample. The slants were allowed to incubate at $39^{\circ} \mathrm{C}$ for $12 \mathrm{wk}$ and colonies were counted at 4,8 , and 12 wk postinoculation.

\section{MAP Confirmation}

A Ziehl-Neelsen acid-fast stain was performed on any sample with a positive GI in BACTEC 12 medium. One drop of Acid Fast Mycohold Cell Adhesive (Wescor Inc., South Logan, UT) was placed onto a microscope slide. About $100 \mu \mathrm{L}$ of sample was added to the slide and mixed thoroughly with the Mycohold. The slides were allowed to air dry and fixed in $100 \%$ methanol for $15 \mathrm{~min}$ at $22^{\circ} \mathrm{C}$. The slides then were stained with a standard Ziehl-Neelsen acid-fast stain followed by methylene blue counter stain.

If samples were positive for acid-fast bacteria, 500 $\mu \mathrm{L}$ of sample was removed from the bottle and added to $100 \mu \mathrm{L}$ of proteinase K $(10 \mathrm{mg} / \mathrm{mL}$; Qiagen Sciences Inc., Germantown, MD). The tubes were incubated at $56^{\circ} \mathrm{C}$ overnight in an orbital water bath and then centrifuged at $15,000 \times g$ for $15 \mathrm{~min}$ at $22^{\circ} \mathrm{C}$ to separate the fractions of the sample. The supernatant was removed, and the pellet was resuspended in $175 \mu \mathrm{L}$ of PBS; DNA was extracted from the pellet using the MagMAX Total Nucleic Acid Isolation Kit (Life Technologies Corp., Carlsbad, CA) on the MagMAX Express machine (Life Technologies Corp.) as directed by the manufacturer.

The extracted DNA was assayed for the presence of MAP by using real-time PCR with the MAP-specific IS900 target. Primer and probe sequences for IS900 were as follows: $200 \mathrm{n} M$ concentration of forward primer 5'-CCGCTAATTGAGAGATGCGATTGG-3', $200 \mathrm{n} M$ concentration of reverse primer $5^{\prime}$-AATCAACTCCAGCAGCGCGGCCTCG- $3^{\prime}$, and $100 \mathrm{n} M$ concentration of probe 5'-TCCACGCCCGCCCAGACAGG-3' with a $5^{\prime}$ fluorescent carboxyfluorescein (FAM) label and a $3^{\prime}$ tetramethylrhodamine (TAMRA) quencher (Integrated DNA Technologies Inc., Coralville, IA). The real-time PCR was conducted in an Applied Biosystems 7500 Fast Real-Time PCR System (Life Technologies Corp.) by using TaqMan Fast Advanced Master Mix (Life Technologies Corp.). Each PCR reaction plate also contained a standard curve that was generated using MAP strain K-10 genomic DNA ( $10 \mathrm{fg} / \mu \mathrm{L}$ to $1 \mathrm{ng} / \mu \mathrm{L}$ ), no-template negative controls, and a positive control consisting of MAP strain 19698 genomic DNA. Amplification conditions were as follows: stage $1: 95^{\circ} \mathrm{C}$ for 20 $\mathrm{s}$; stage 2: $94^{\circ} \mathrm{C}$ for $3 \mathrm{~s}$ and $66^{\circ} \mathrm{C}$ for $30 \mathrm{~s}$, repeated for 40 cycles. All samples with a threshold cycle $\left(\mathbf{C}_{\mathbf{t}}\right)$ less than or equal to 37 were considered positive.

Mycobacterium avium ssp. paratuberculosis growth was also confirmed on the HEY medium slants by PCR as stated above by placing suspect colonies into the beadbeat tube from the MagMAX Total Nucleic Acid Identification Kit and following the manufacturer's instructions. A sample was classified as positive or nega- 
tive only if both the acid-fast stain and PCR results confirmed the GI or the presence of colonies. A sample was considered contaminated if acid-fast organisms were present in the smear but the sample was negative for the IS900 gene.

\section{Direct PCR Detection}

All milk samples that were subject to culture were also used for direct PCR detection. Direct PCR was performed on 54 additional samples that were not cultured due to lack of sufficient volume for both tests. Additionally, the number of colony-forming units of MAP per milliliter of milk was extrapolated from a standard curve generated by experimental inoculation of negative milk with serial dilutions of MAP strain 167. Triplicate samples of $18 \mathrm{~mL}$ of milk and $2 \mathrm{~mL}$ of the respective dilution of bacterial suspension were prepared to yield final MAP concentrations of $10^{2}, 10^{4}, 10^{6}$, and $10^{8} \mathrm{cfu} / \mathrm{mL}$ of milk. Each dilution was subjected to the basic milk DNA extraction protocol as previously described (Bradner et al., 2013) and $\mathrm{C}_{t}$ values were plotted against MAP concentration to generate a standard curve.

The milk sample $(10 \mathrm{~mL})$ was transferred to a sterile tube and centrifuged at $5,600 \times g$ for $30 \mathrm{~min}$ at $4^{\circ} \mathrm{C}$. The whey layer was removed, and the remaining cream and pellet were resuspended in $800 \mu \mathrm{L}$ of PBS. As much of the solution as possible was transferred to a sterile 1.5 -mL tube containing $200 \mu \mathrm{L}$ of $10 \mathrm{mg} / \mathrm{mL}$ proteinase $\mathrm{K}$. The samples were incubated at $56^{\circ} \mathrm{C}$ for $1 \mathrm{~h}$ and then centrifuged at $15,000 \times g$ for $15 \mathrm{~min}$ at $22^{\circ} \mathrm{C}$. The liquid fraction was removed, and the cream and pellet were resuspended in $200 \mu \mathrm{L}$ of PBS. A volume of 175 $\mu \mathrm{L}$ of this solution was transferred to a MagMAX Total Nucleic Acid Isolation Kit bead beat tube (Life Technologies Corp.), and the DNA was extracted on the MagMAX Express particle processor (Life Technologies Corp.) per the manufacturer's instructions.

Polymerase chain reaction targeting the IS900 gene was performed as before except using the TaqMan Environmental Master Mix 2.0 (Life Technologies Corp.). Amplification conditions were as follows: stage 1: $95^{\circ} \mathrm{C}$ for $10 \mathrm{~min}$; stage $2: 94^{\circ} \mathrm{C}$ for $25 \mathrm{~s}$ and $66^{\circ} \mathrm{C}$ for $1 \mathrm{~min}$, repeated for 45 cycles. All samples with a $\mathrm{C}_{\mathrm{t}}$ less than or equal to 42 were considered positive.

\section{Statistical Analysis}

Statistical analysis was performed using JMP software (version 8; SAS Institute Inc., Cary, NC). The frequency distribution of positive samples within infection group and by method of detection, and the status of cows after calving was analyzed by chi-squared analysis with the Fisher exact test. Data summarizing MAP shedding by infection group were analyzed by one-way ANOVA analysis. When significant effects $(P<0.05)$ due to treatment group were present, least squares means separation was computed using the Tukey Kramer post hoc test.

\section{RESULTS}

Results of culture and PCR on milk and colostrum samples collected from cows during the 305-d lactation cycle are presented in Table 1 . The total number of milk samples assayed by direct PCR was greater than that by culture due to a lack of necessary sample volume for some collection dates. The presence of MAP was detected most frequently $(P<0.01)$ in milk samples that were obtained from cows in the clinical stage of disease compared with cows that were subclinically infected, regardless of detection method. Direct PCR was more sensitive $(P<0.01)$ than either liquid or HEY culture medium for detection of the presence of MAP in either colostrum or milk samples. Samples collected from clinical cows during the lactation cycle were $49.2 \%$ positive by direct PCR compared with 18.4 and $12.9 \%$ positive by culture on liquid and HEY mediums, respectively (Figure 1). Although a greater number of milk samples (293 samples by culture and 317 samples by direct PCR) were processed from subclinically infected cows, MAP was detected with lower frequency, regardless of detection method (Table 1). The highest level of MAP recovery from milk samples obtained from subclinical cows was achieved by direct PCR, with $12.6 \%$ of samples positive (Figure 1). None of the samples from the control cows were positive by any of the detection methods used in this study. Over the course of the 305-d lactation cycle, 29 of the 35 lactations from clinical cows and 26 of the 48 lactations from subclinical cows were positive for MAP using PCR as the detection method.

Culture methods were less sensitive than PCR, reducing the rate of detection to 13 of 30 lactations from clinical cows and 8 of 44 lactations from subclinical cows when using either method of culture to detect MAP in the milk samples. Viable MAP was shed primarily into milk and colostrum in early lactation (DIM 0-60) and less frequently shed in mid (DIM 60-240) and late (DIM 240-305) lactation (Table 1). All of the culture positive milk and colostrum samples from cows with clinical JD were collected in early lactation. Likewise, 10 of 12 culture-positive milk and colostrum samples from subclinical cows were collected in early lactation and only 2 positive samples were collected in mid lactation. This trend was consistent for detection of the presence of MAP in milk and colostrum samples 
Table 1. Shedding pattern of Mycobacterium avium ssp. paratuberculosis in milk and colostrum of naturally infected dairy cows during the 305-d lactation period

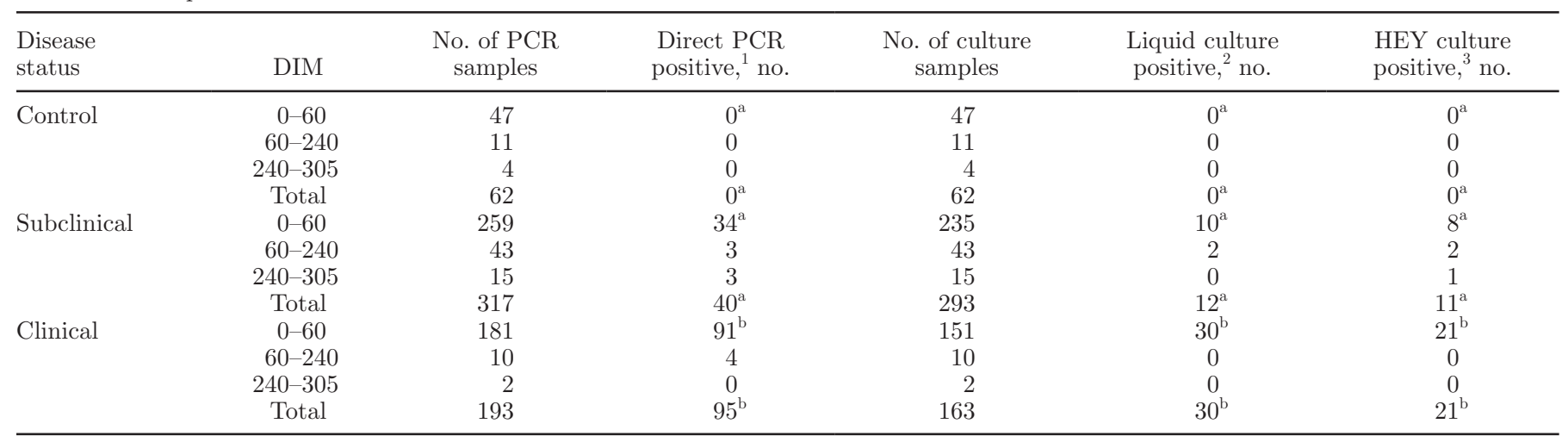

$\overline{\mathrm{a}, \mathrm{b}}$ Different superscript letters within a column denote significance between treatment groups within DIM for each detection method $(P<0.01)$. ${ }^{1}$ Direct PCR was performed for the IS900 target gene.

${ }^{2}$ Liquid culture refers to samples cultured in BACTEC 12B (Becton Dickinson, Franklin Lakes, NJ) or para-JEM (Thermo Fisher Scientific, Trek Diagnostic Systems Inc., Cleveland, OH) media.

${ }^{3} \mathrm{HEY}=$ Herrold's egg yolk medium.

by direct PCR as well. Of the positive samples obtained from clinical cows, 91 of 95 were detected by direct PCR in early lactation and 4 of 95 were detected in mid lactation (Table 1). Similarly, 34 of the 40 positive samples collected from subclinical cows were obtained in early lactation, 3 in mid lactation, and 3 in late lactation.

Semiquantitative results for amount of MAP shed in the milk of naturally infected cows were obtained from both PCR and liquid culture. Culture results using

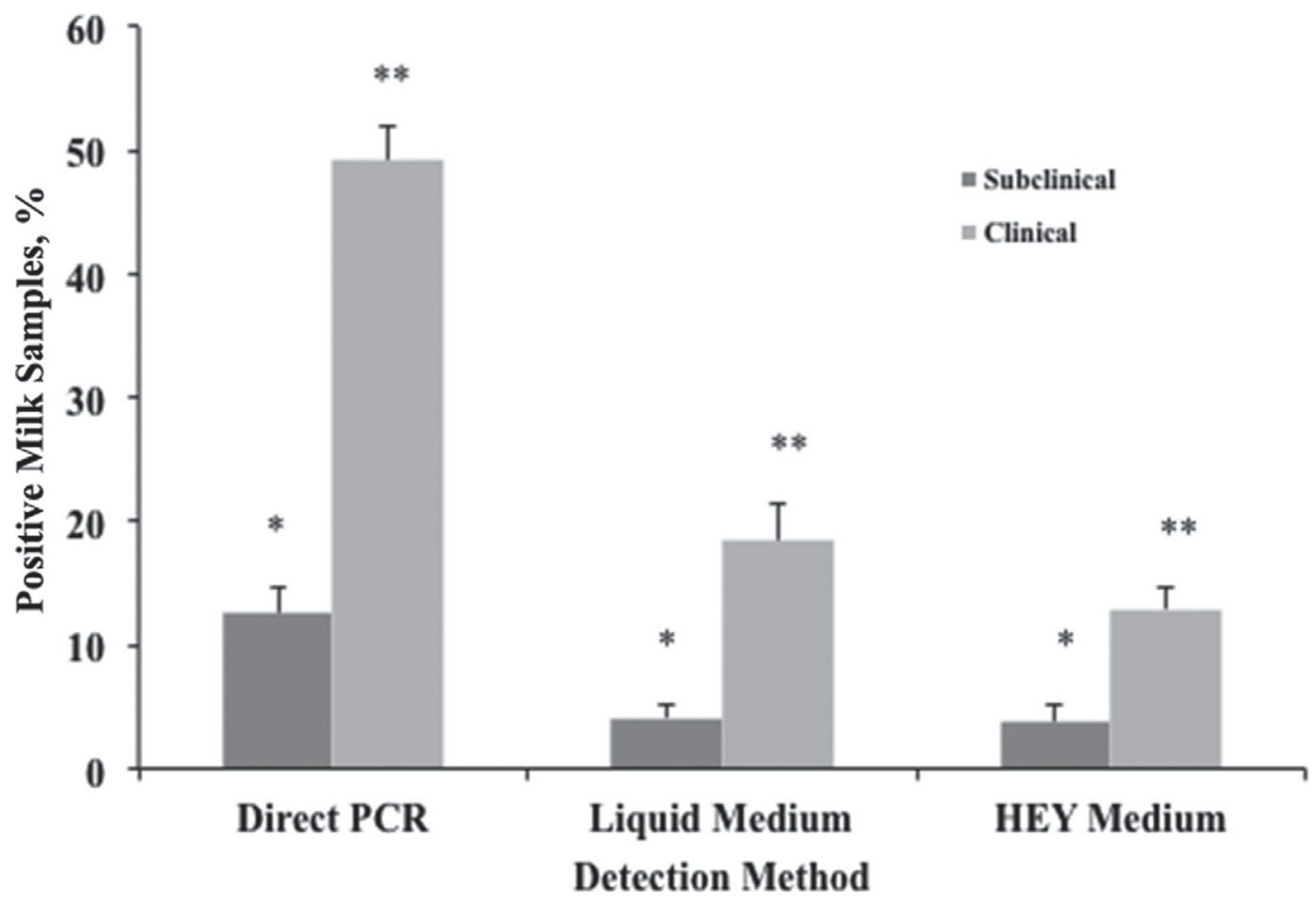

Figure 1. Percentage of positive milk and colostrum samples from cows with subclinical and clinical Mycobacterium avium ssp. paratuberculosis infection as determined by direct PCR for the IS900 target gene, and by culture on liquid medium [BACTEC 12B (Becton Dickinson, Franklin Lakes, NJ) or para-JEM (Thermo Fisher Scientific, Trek Diagnostic Systems Inc., Cleveland, OH)] and Herrold's egg yolk medium $(\mathrm{HEY})$. Data analysis was performed by chi-squared analysis. ${ }^{*}$ and $* *$ indicate that differences between subclinical and clinical treatment groups were significant $(P<0.01)$ within method. Data are presented as mean percentage of positive milk samples \pm SEM. 


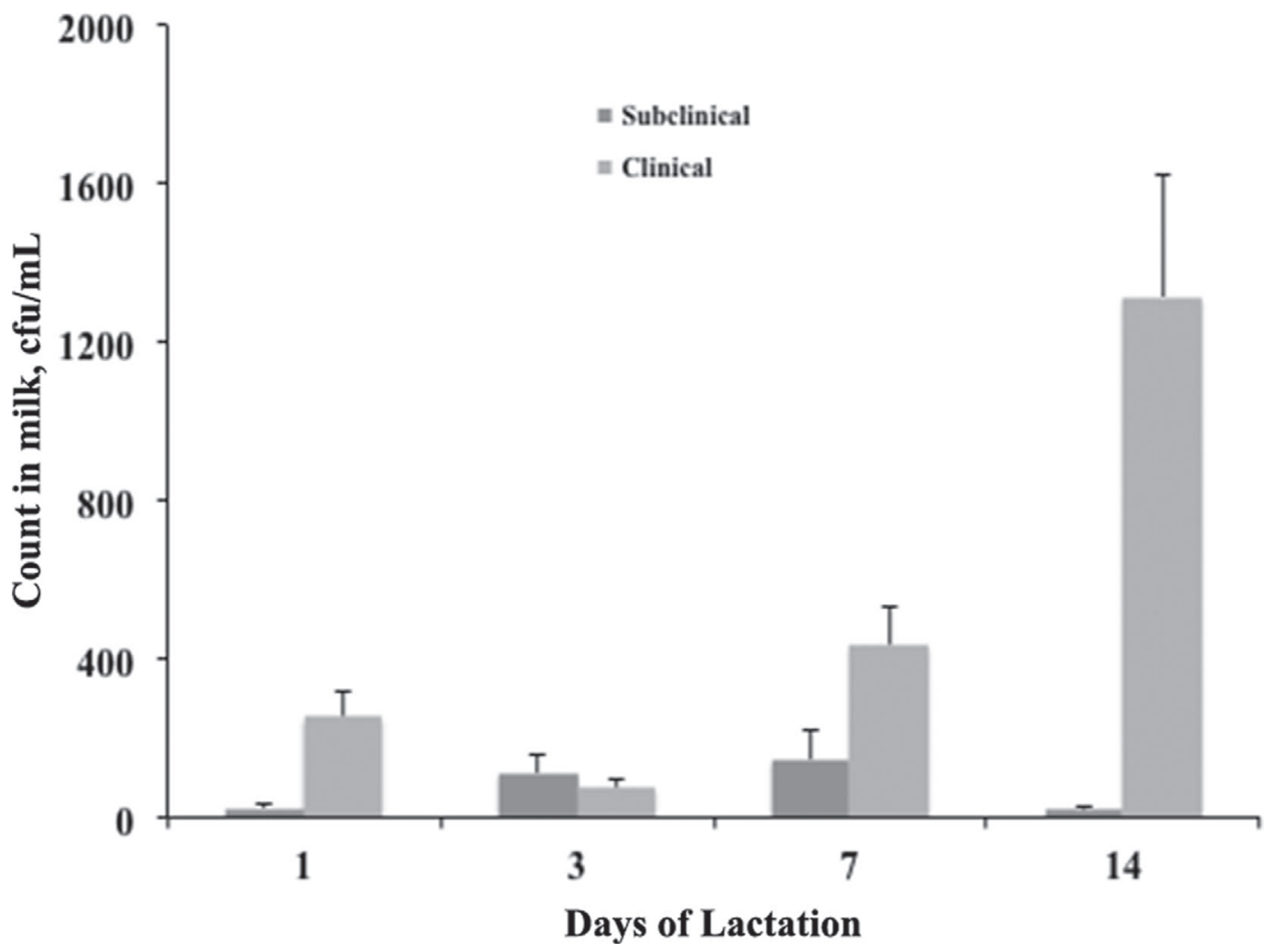

Figure 2. Semiquantitative shedding (cfu/mL) of Mycobacterium avium ssp. paratuberculosis in colostrum and milk of cows in subclinical and clinical infection on d 1,3, 7, and 14 of lactation as determined by direct PCR for the IS900 target gene. Data are presented as mean percentage of positive milk samples \pm SEM.

HEY medium were sporadic and insensitive, yielding very low colony counts, so are not presented. Results obtained by PCR demonstrated that the number of MAP shed in colostrum (d 1) was higher for clinical cows than subclinical cows at $254 \pm 63(\mathrm{n}=16)$ and 24 $\pm 12 \mathrm{cfu} / \mathrm{mL}(\mathrm{n}=4)$, respectively (Figure 2$)$. A second peak of shedding was noted for clinical cows at d 14 postcalving, with a mean value of $1,316 \pm 310 \mathrm{cfu} / \mathrm{mL}$ of milk $(\mathrm{n}=18)$, an effect that was primarily due to 1 high shedder $(21,289 \mathrm{cfu} / \mathrm{mL})$. The number of positive samples were stratified somewhat equally during the first 2 wk of lactation, with 4, 6, 4, and 9 samples from subclinical cows and 16, 20,20, and 18 samples from clinical cows PCR positive on d 1, 3, 7, and 14, respectively, suggesting that posttransition milk may also be a key period for MAP shedding. A similar profile of MAP shedding was obtained for colostrum samples (d 1) with liquid culture, with values averaging $474 \pm$ $266(\mathrm{n}=7)$ and $31 \pm 24 \mathrm{cfu} / \mathrm{mL}$ of colostrum ( $\mathrm{n}=$ 4 ; data not shown). Although calculated numbers of colony-forming units per milliliter of colostrum or milk did not correlate precisely between PCR and liquid culture methods of quantification, the trends noted in PCR results were paralleled in liquid culture. Colos- trum collected from clinical dams had higher levels of MAP compared with subclinical cows and a secondary shedding peak was noted and sustained on $\mathrm{d} 7$ and 14 of lactation for milk from clinical cows. Variability was high within the treatment groups due to the limited number of positive samples at each sampling time point, thereby precluding any statistical significance between treatments.

Of the 29 clinical cows, 19 died from JD within 1 yr of calving (Table 2). Samples collected from these cows accounted for 26 of the 30 milk samples that were culture positive in liquid media, and 17 of the 21 milk samples that were culture positive on HEY medium. In addition, 66 of the 95 milk samples that were positive for MAP via direct PCR were collected from this group of clinical cows.

\section{DISCUSSION}

This study investigated the shedding of MAP into milk and colostrum from naturally infected dairy cows over the 305-d lactation cycle. The results indicated a strong correlation between the shedding of MAP into the milk and colostrum and stage of disease. With more 
Table 2. Contingency analysis of milk and colostrum samples collected from clinical cows in the study ${ }^{1}$

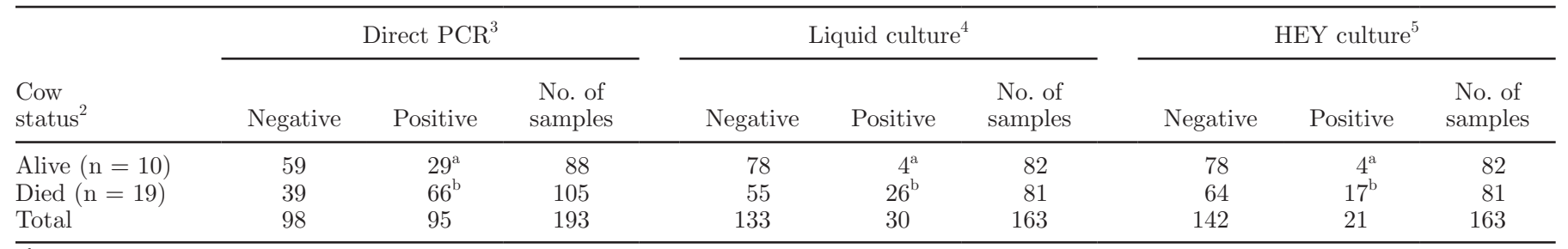

${ }^{\mathrm{a}, \mathrm{b}}$ Different superscript letters within a column denote significance between treatment groups for each detection method $(P<0.01)$.

${ }^{1}$ Samples are classified as positive or negative for detection of Mycobacterium avium ssp. paratuberculosis by direct PCR targeting the IS900 gene or culture.

${ }^{2}$ Clinical cows were stratified as to whether they survived the lactation period (alive; $\mathrm{n}=10$ ) or succumbed to Johne's disease within 1 yr (died; $\mathrm{n}=19$ ) of the calving period from which samples were collected.

${ }^{3}$ Direct PCR was performed for the IS900 target gene.

${ }^{4}$ Liquid culture refers to samples cultured in BACTEC 12B (Becton Dickinson, Franklin Lakes, NJ) or para-JEM (Thermo Fisher Scientific, Trek Diagnostic Systems Inc., Cleveland, OH) media.

${ }^{5} \mathrm{HEY}=$ Herrold's egg yolk medium.

advanced MAP infection, the likelihood increased for MAP detection by PCR or isolation of viable MAP by culture. Mycobacterium avium ssp. paratuberculosis was detected by direct PCR in 49.2 and $12.6 \%$ of samples collected from cows in the clinical and subclinical stages of JD, respectively. Similarly, MAP was detected by culture in the liquid medium in 18.4 and $4.1 \%$ of samples collected from cows in the clinical and subclinical stages of JD, respectively.

One possible model for the dissemination of MAP as JD progresses is the migration of intestinal macrophages containing MAP to the mesenteric lymph nodes, leading to the possibility of hematogenous or lymphatic spread of MAP. This spread of MAP allows it to infiltrate milk, blood, semen, and other extraintestinal tissues, such as the mammary lymph nodes (Sweeney et al., 1992; Koenig et al., 1993; Chiodini, 1996; Ayele et al., 2004). One study found that the proportion of culture-positive milk samples and supramammary lymph nodes was higher in cows that were heavily shedding MAP into their feces than cows that were classified as intermediate or light shedders (Sweeney et al., 1992). To further support this finding, a recent study examined lymph fluid collected from the udder of clinical, subclinical, and negative cows from herds with a high prevalence of JD (Khol et al., 2012). The lymph fluid was positive for the MAP-specific gene target $I S 900$ by PCR, with increasing occurrence in samples collected from clinical cows (66.7\%), compared with cows that were subclinically infected $(42.8 \%)$ and negative $(38.7 \%)$.

In the present study, the number of milk samples determined to be positive for MAP was much higher by direct PCR than the number detected by culture, regardless of whether they were collected from cows with clinical or subclinical JD. This discrepancy can be explained by the fact that PCR can detect both live and dead bacteria, thereby increasing the likelihood of detection, as intracellular MAP may have been killed by macrophages within the mammary gland. Second, direct PCR has a lower detection threshold than either culture method used in the present study. Previous experiments conducted in our laboratory comparing the efficacy of PCR and culture demonstrated a sensitivity of detection of less than $10^{2} \mathrm{cfu} / \mathrm{mL}$ for liquid medium and $10^{4} \mathrm{cfu} / \mathrm{mL}$ for HEY medium, compared with less than $10 \mathrm{cfu} / \mathrm{mL}$ for direct PCR (Bradner et al., 2013). Culture is beneficial because it provides information on the presence of live, infectious bacteria present in milk samples but it also has a higher detection threshold due to methods used to process the samples. Milk samples must be decontaminated before culture due to the presence of confounding microorganisms, including other bacteria, molds, and fungi. However, decontamination procedures applied to the milk are potentially lethal to MAP as well (Dundee et al., 2001; Burdz et al., 2003). In addition, the decontamination and processing of milk samples before culture may result in loss of MAP due to centrifugation, partitioning of the sample, and dilution factors. Polymerase chain reaction provides more information because a positive result may be indicative of the presence of either whole or fragmented MAP cells. Both of these are important, as even fragmented nonviable MAP can elicit immune responses when ingested, leading to inflammatory reactions within the host (Hines et al., 2014). Detection by PCR is also advantageous, as it allows for same day acquisition of results compared with 12 wk to obtain results from culture.

Viable MAP was isolated primarily from milk and colostrum in early lactation. This finding can be explained, in part, by differences in milk and colostrum 
composition. Milk fat can be as high as $6 \%$ at the beginning of lactation and then decreases to around $3.7 \%$ at around $10 \mathrm{~d}$ postcalving (Spreer, 1998). Mycobacterium avium ssp. paratuberculosis preferentially partitions into the cream fraction of milk, so it is possible that MAP will be more easily shed into milk with a higher fat content (Gao et al., 2005). Additionally, during the dry period, the percentage of mononuclear macrophages increases from 8 to $29 \%$ in the mammary gland from $\mathrm{d} 1$ of the dry period to between -2 and 0 d before parturition (Hurley et al., 1990). If this influx of macrophages into the mammary gland contained macrophages infected with MAP, it could result in the introduction of a higher concentration of MAP into the colostrum. Thereby, the physiologic events that normally occur at parturition could be responsible for the increased detection of MAP in samples obtained during early lactation.

When examining the results for MAP detection on an individual-cow basis, some interesting findings became apparent for cows that were classified as clinical. Of the 29 clinical cows that were sampled, 19 of these cows died within $1 \mathrm{yr}$ of calving. These lactations accounted for $87 \%$ of the culture-positive samples and $70 \%$ of the direct PCR-positive samples for clinical cows. These findings are consistent with what would be expected, as dissemination of MAP throughout the body and shedding of MAP into the feces increases as JD progresses (Chiodini et al., 1984; Valentin-Weigand and Goethe, 1999). Therefore, it appears the frequency and amount of MAP shed into milk and colostrum increases continuously until the animal succumbs to JD.

Interestingly, MAP was isolated from the colostrum and milk of a first-lactation subclinical cow on d 1, 3, and 7 after calving. This result was contrary to results from the other milk samples collected from subclinical cows. It is difficult to speculate why this young cow was shedding MAP into the milk so consistently. $M y$ cobacterium avium ssp. paratuberculosis shedding into the milk was sporadically detected for other subclinical cows, as would be expected in the early stages of MAP infection, as MAP shed into the feces is intermittent (Whitlock and Buergelt, 1996).

Unfortunately, very few samples were obtained from mid and late lactation from cows in the clinical stage of JD, primarily because the animals succumbed to JD in the early days of lactation. It would have been interesting to see if MAP was steadily shed into the milk throughout the complete lactation cycle, whether shedding was intermittent similar to fecal shedding, or whether MAP shedding continued to increase as JD progressed. However, for accurate assessment to obtain a better understanding of when and how MAP is shed into milk and colostrum, it may be necessary to develop more sensitive detection methods.

In summary, this study definitively demonstrates that MAP is shed into milk and colostrum, preferentially by cows in advanced stages of JD and in early lactation. This demonstration proves that dam-to-calf transmission of MAP by milk consumption is a viable route of transmission. More importantly, research has found that exposure of MAP to high-osmolarity environments, such as those found in bovine mammary epithelial cells and milk, causes a change in the gene expression in MAP that is associated with an invasive phenotype (Patel et al., 2006). A meta-analysis of studies found that calves less than 6 mo of age had a $74 \%$ chance of becoming infected upon exposure to MAP, with likelihoods decreasing to $50 \%$ between 6 to 12 mo of age and $19.3 \%$ in animals older than 12 mo (Windsor and Whittington, 2010). Therefore, if a newborn calf is allowed to suckle even once, it is experiencing the highest likelihood of infection by MAP. Additionally, if producers feed pooled colostrum, many calves could be at risk of infection by MAP. Veterinarians now have critical information to educate dairy producers on the risk of MAP transmission through milk and colostrum and the need to change calf-rearing practices to accommodate these findings.

\section{ACKNOWLEDGMENTS}

We thank Philip Dykema, Robin Swanson, and Hannah Wilson of the Mycobacteria and Brucella Section, National Veterinary Services Laboratories (USDAAPHIS, Ames, IA) for their training, advice, and assistance. We also thank Erica Hellmich and Margaret Walker of the Johne's Disease Research Project (USDAARS, NADC, Ames, IA) for their technical assistance in the laboratory.

\section{REFERENCES}

Ayele, W. Y., M. Bartos, P. Svastova, and I. Pavlik. 2004. Distribution of Mycobacterium avium subsp. paratuberculosis in organs of naturally infected bull-calves and breeding bulls. Vet. Microbiol. 103:209-217.

Bradner, L., S. Robbe-Austerman, D. C. Beitz, and J. R. Stabel. 2013. Chemical decontamination with $N$-acetyl-L-cysteine-sodium hydroxide improves recovery of viable Mycobacterium avium subsp. paratuberculosis organisms from cultured milk. J. Clin. Microbiol. 51:2139-2146.

Burdz, T. V. N., J. Wolfe, and A. Kabani. 2003. Evaluation of sputum decontamination methods for Mycobacterium tuberculosis using viable colony counts and flow cytometry. Diagn. Microbiol. Infect. Dis. 47:503-509.

Chiodini, R. J. 1996. Immunology: Resistance to paratuberculosis. Vet. Clin. North Am. Food Anim. Pract. 12:313-343.

Chiodini, R. J., H. J. Van Kruiningen, and R. S. Merkal. 1984. Ruminant paratuberculosis (Johne's disease): The current status and future prospects. Cornell Vet. 74:218-262. 
Dundee, L., I. R. Grant, H. J. Ball, and M. T. Rowe. 2001. Comparative evaluation of four decontamination protocols for the isolation of Mycobacterium avium subsp. paratuberculosis from milk. Lett. Appl. Microbiol. 33:173-177.

Gao, A., J. Odumeru, M. Raymond, and L. Mutharia. 2005. Development of improved method for isolation of Mycobacterium avium subsp. paratuberculosis from bulk tank milk: effect of age of milk, centrifugation, and decontamination. Can. J. Vet. Res. 69:81-87.

Hines, M. E., II, S. E. Turnquist, M. R. S. Ilha, S. Rajeev, A. L. Jones, L. Whittington, J. P. Bannantine, R. G. Barletta, Y. T. Gröhn, R. Katani, A. M. Talaat, L. Li, and V. Kapur. 2014. Evaluation of novel oral vaccine candidates and validation of a caprine model of Johne's disease. Front. Cell. Infect. Microbiol. 4:26.

Hurley, D. J., M. H. Kensinger, A. M. Mastro, and R. A. Wilson. 1990. An evaluation of the mononuclear cells derived from bovine mammary gland dry secretions using leukocyte antigen specific monoclonal antibodies, light scattering properties and non-specific esterase staining. Vet. Immunol. Immunopathol. 25:177-193.

Khalifeh, M. S., A. M. Al-Majali, and J. R. Stabel. 2009. Role of nitric oxide production in dairy cows naturally infected with Mycobacterium avium subsp. paratuberculosis. Vet. Immunol. Immunopathol. 131:97-104.

Khol, J. L., P. J. Pinedo, C. D. Buergelt, L. M. Neumann, W. Baumgartner, and D. O. Rae. 2012. The collection of lymphatic fluid from the bovine udder and its use for the detection of $\mathrm{Myco-}$ bacterium avium subsp. paratuberculosis in the cow. J. Vet. Diagn. Invest. 24:23-31.

Koenig, G. J., G. F. Hoffsis, W. P. Shulaw, S. Bech-Nielsen, D. M. Rings, and G. St-Jean. 1993. Isolation of Mycobacterium paratuberculosis from mononuclear cells in tissues, blood, and mammary glands of cows with advanced paratuberculosis. Am. J. Vet. Res. 54:1441-1445.

Nielsen, S. S., and N. Toft. 2012. Effect of days in milk and milk yield on testing positive in milk antibody ELISA to Mycobacterium avium subsp. paratuberculosis in dairy cattle. Vet. Immunol. Immunopathol. 149:6-10.
Ott, S. L., S. J. Wells, and B. A. Wagner. 1999. Herd-level economic losses associated with Johne's disease on US dairy operations. Prev. Vet. Med. 40:179-192.

Patel, D., L. Danelishvili, Y. Yamazaki, M. Alonso, M. L. Paustian, J. P. Bannantine, L. Meunier-Goddik, and L. E. Bermudez. 2006. The ability of Mycobacterium avium subsp. paratuberculosis to enter bovine epithelial cells is influenced by preexposure to a hyperosmolar environment and intracellular passage in bovine mammary epithelial cells. Infect. Immun. 74:2849-2855.

Pithua, P., S. M. Godden, S. J. Wells, and M. J. Oakes. 2009. Efficacy of feeding plasma-derived commercial colostrum replacer for the prevention of transmission of Mycobacterium avium subsp paratuberculosis in Holstein calves. J. Am. Vet. Med. Assoc. 234:11671176.

Spreer, E. 1998. Milk as a raw material and food. Pages 11-58 in Milk and Dairy Product Technology. Marcel Dekker Inc., New York, NY.

Stabel, J. R. 2008. Pasteurization of colostrum reduces the incidence of paratuberculosis in neonatal dairy calves. J. Dairy Sci. 91:36003606.

Sweeney, R. W., R. H. Whitlock, and A. E. Rosenberger. 1992. Mycobacterium paratuberculosis cultured from milk and supramammary lymph nodes of infected asymptomatic cows. J. Clin. Microbiol. 30:166-171.

Valentin-Weigand, P., and R. Goethe. 1999. Pathogenesis of Mycobacterium avium subspecies paratuberculosis infections in ruminants: Still more questions than answers. Microbes Infect. 1:1121-1127.

Whitlock, R. H., and C. D. Buergelt. 1996. Preclinical and clinical manifestations of paratuberculosis (including pathology). Vet. Clin. North Am. Food Anim. Pract. 12:345-356.

Windsor, P. A., and R. J. Whittington. 2010. Evidence for age susceptibility of cattle to Johne's disease. Vet. J. 184:37-44.

Zervens, L. M.-L., S. S. Nielsen, and G. Jungersen. 2013. Characterization of an ELISA detecting immunoglobulin G to Mycobacterium avium subsp. paratuberculosis in bovine colostrum. Vet. J. 197:889-891. 